\title{
Correction to: Hierarchical Control for the One-dimensional Plate Equation with a Moving Boundary
}

\author{
I. P. de Jesus ${ }^{1}$ - J. Limaco ${ }^{2} \cdot$ M. R. Clark ${ }^{1}$
}

Published online: 22 August 2018

(C) Springer Science+Business Media, LLC, part of Springer Nature 2018

\section{Correction to: Journal of Dynamical and Control Systems https://doi.org/10.1007/s10883-018-9413-z}

The original version of this article unfortunately contained a mistake. On page 653, Equation (5.2) should be

$$
\begin{aligned}
& \varphi^{\prime \prime}+L^{*} \varphi=\gamma(t) \psi \text { in } Q, \\
& \psi^{\prime \prime}+L \psi=0 \text { in } Q, \\
& v^{\prime \prime}+L v=0 \text { in } Q, \\
& p^{\prime \prime}+L^{*} p=\gamma(t)\left(v-v_{2}\right) \text { in } Q, \\
& \varphi=0 \text { on } \Sigma, \\
& \varphi_{y}=0 \text { on } \Sigma, \\
& \psi=0 \text { on } \Sigma, \\
& \psi_{y}=\left\{\begin{array}{l}
0 \text { on } \Sigma_{1}, \\
\frac{a(t)}{\sigma} \varphi_{y y} \text { on } \Sigma_{2}, \\
0 \backslash \Sigma_{0}, \\
v_{y}=\left\{\begin{array}{l}
a(t) \varphi_{y y} \text { on } \Sigma_{1}, \\
0 \text { on } \Sigma \backslash \Sigma_{0 y},
\end{array}\right. \\
v=0 \text { on } \Sigma, \\
p=0 \text { on } \Sigma, \\
p_{y}=0 \text { on } \Sigma, \\
\varphi(., T)=f^{0}, \varphi^{\prime}(., T)=f^{1} \text { in } \Omega, \\
v(0)=v^{\prime}(0)=0 \text { in } \Omega, \\
p(T)=p^{\prime}(T)=0 \text { in } \Omega .
\end{array}\right.
\end{aligned}
$$

The online version of the original article can be found at https://doi.org/10.1007/s10883-018-9413-z.

I. P. de Jesus

isaias@ufpi.edu.br

J. Limaco

jlimaco@vm.uff.br

M. R. Clark

marcondesclark@ufpi.edu.br

1 Universidade Federal do Piauí, DM, Teresina, PI, Brazil

2 Universidade Federal Fluminense, IME, Niterói, RJ, Brazil 